\title{
THE TEXAS RADIO ASTROMETRIC SURVEY
}

JAMES N. DOUGLAS

University of Texas, Austin, Tex., U.S.A.

\begin{abstract}
The 5-element Texas Interferometer is engaged in a $365 \mathrm{MHz}$ survey of the sky with a primary goal of establishing positions of about 50000 discrete radio sources with an accuracy of about 1 " in each coordinate. Measurements are made relative to optical positions of identified sources, and the 2000 positions thus far obtained support our expectations of the accuracy of the survey. In a companion program, optical positions of objects near radio source positions are being measured to $\pm \frac{1}{2}$ " accuracy on glass copies of the Palomar Sky Survey, yielding both improved overall calibration of the radio positions and identification of associated optical objects on the basis of position coincidence alone, without the selection effects usually introduced by auxiliary identification criteria.
\end{abstract}

